\title{
¿Qué es un dispositivo?
}

\author{
Óscar Moro abadia* \\ Departamento de Ciencias Históricas \\ Universidad de Cantabria \\ papitu2000@hotmail.com
}

\section{INTRODUCCIÓN}

Los días 24 y 25 de abril de 1998 se celebró en l'Université de Louvain-LaNeuve un congreso internacional que, bajo el título «Dispositifs et médiation des savoirs" ', trataba de ofrecer algunas respuestas a una pregunta planteada por Gilles Deleuze diez años atrás: ¿Qué es un dispositivo? (Deleuze 1989).

La pertinencia, entonces y ahora, de dicha cuestión obedecía a la paradoja nacida de una doble constatación: Por un lado, la notable difusión del término «dispositivo» en el campo de las ciencias humanas y, por otro, los escasos ejercicios de reflexión teórica sobre su significado. En este sentido y teniendo en cuenta que dicho término ha sido utilizado especialmente en trabajos de sociología cualitativa, el marco que ofrece una revista sobre metodología de las ciencias sociales parece el lugar idóneo para retomar la cuestión y plantear algunas soluciones.

Efectivamente, desde que Michel Foucault (1926-1984) hablase del «dispositivo disciplinario» (Foucault 1975: 173) o del «dispositivo de la sexualidad»

* Este artículo nació de una conversación que mantuve con el profesor Hubert L. Dreyfus durante una estancia en la Universidad de Berkeley. Muchas de las ideas aquí expuestas recogen alguna de sus sugerencias y, en este sentido, quisiera expresarle mi agradecimiento. Tampoco quisiera olvidarme de algunos compañeros y amigos que han sido una ayuda inestimable en la redacción definitiva de este ensayo: Carlos Nieto, Angel Pelayo, Manuel R. González Morales y Jorge González.

1 Congreso Internacional «Dispositifs et méditation dans des savoirs organizado por el GReMS (Groupe de Recherche en Mediations des Savoirs-Université catholique de Louvain (Belgique)), en colaboración con el GRAME (Groupe de Recherches sur les Apprentissages, les Médias et l'Education Université de Paris 8). 
(Foucault 1976: 99) hemos asistido a la proliferación de trabajos que desde perspectivas tan diferentes como la filosofía, el derecho, la pedagogía o el feminismo, hablan de «dispositivos» carcelarios, pedagógicos, televisivos e incluso funerarios (Vandendorpe 1999). Como acabo de señalar y como me propongo repasar en esta introducción, dicha proliferación ha sido especialmente importante en el caso de la sociología.

En primer lugar, la idea de estudiar las relaciones entre saber y poder dentro del espacio de un dispositivo ha adquirido gran protagonismo en sociología de la ciencia, tal y como demuestran los trabajos de Steven Shapin, Ian Hacking y Bruno Latour entre otros ${ }^{2}$. Todos ellos han incidido en el análisis de la producción, circulación y recepción del saber científico/ técnico a través de lo que nosotros denominamos «dispositivos».

El concepto también ha sido utilizado recurrentemente en el campo de la sociología del trabajo (p.e. Bessy 1997: 35, Eymard-Duvernay 1997: 17) donde se ha planteado desde la importancia de analizar «dispositivos técnicos sofisticados o que hacen un uso importante de útiles informáticos de comunicación, permitiendo a grupos dispersos trabajar juntos en un grupo-proyecto,» ${ }^{3}$ (Cardon 1997: 5) hasta la necesidad de considerar que «toda práctica tiene lugar en un dispositivo que comprende objetos materiales [...] en un espacio organizado socialmente y bajo la regulación de discurso.» (Poitou 1980: 95) ${ }^{4}$ Lo mismo podría decirse de la sociología de los medios de comunicación donde, desde que en 1971 Schaeffer definiera las relaciones entre los profesionales de la comunicación y el público como un «dispositivo estratégico» ${ }^{5}$ (Schaeffer 1971: 61), se ha venido hablando de «dispositivos televisuales» (Nel 1998), de una «edad de oro de los dispositivos» (Flageul 1999) o de un «dispositivo que concierne únicamente a la proyección [cinematográfica] y en la que está incluido el sujeto al que se dirige dicha proyección» ${ }^{6}$ (Baudry 1975: 58).

Tan prolífica utilización contrasta con el escaso número de «enfoques realmente reflexivos, abstractos, de la noción de dispositivo. Habitualmente, el término [...] aparece sin verdadera definición, modestamente enrollado en el cora-

${ }^{2}$ Frente a la epistemología que estudia «la ciencia ya constituida» ( $\ll$ Ready Made Science»), Bruno Latour reivindica una sociología de la ciencia que estudie «la ciencia que está haciéndose» ( Science in the Making») (Latour 1987). Privilegiando el análisis de la investigación científica, i.e. de la praxis científica, Latour ha puesto sobre la mesa la existencia de «extraordinarios dispositivos de producción del saber y, en verdad, de mediación del saber» («Extraordinaires dispositifs de production du savoir, et en vérité, de médiation du savoir», Berten 1999: 33). Un buen ejemplo en su libro Laboratory Life donde, a partir de una metodología antropológica, los autores analizan la vida en un laboratorio científico apoyándose en ideas que remiten a la definición foucaultiana de dispositivo (Latour \& Woolgar 1979).

${ }_{3}$ «Dispositifs techniques sophistiqués ou qui font un usage important d'outils informatiques de communication, permettant à des équipes dispersées de travailler ensemble en groupe-projet».

${ }_{4}^{4}$ «Toute pratique s'accomplit dans un dispositif qui comprend des objets matériels [...] dans un espace organisé socialement et sous la régulation de discours».

5 "Dispositif stratégique».

6 «Dispositif qui concerne uniquement la projection et dans lequel le sujet à qui s'adresse la projection est inclus». 
zón de una frase o de una expresión más boyante» ${ }^{7}$ (Belin 1999: 251). Parece «como si su carácter de operatividad dispensase de tener que pensarlo en su pertinencia teórica.» ${ }^{8}$ (Jacquinot-Delaunay \& Monnoyer 1999: 9). En mi opinión, dos razones explican tan escasa reflexión teórica: en primer lugar la poligénesis del concepto (que invalida la extendida creencia de su origen foucaultiano) ${ }^{9} \mathrm{y}$, en segundo lugar, su gran plasticidad que ha acabado por convertirle en una palabra-maleta que «permite explicar de manera elegante y concisa lo que de otro modo exigiría el empleo de perífrasis azarosas» ${ }^{10}$ (Belin 1999: 252).

Por todo ello, el objetivo de este artículo es sugerir una reflexión teórica sobre el concepto dispositivo que permita, en la medida de nuestras posibilidades, reducir la mencionada distancia que separa su definición y su uso. Dado el carácter poligenético y maleable del término, existen dos posibilidades para llevar a cabo esta tarea: Proceder a una genealogía temática que permita comprender la génesis del concepto en cada disciplina en la que se ha aplicado ${ }^{11}$, o examinar la referencia a la que remiten la mayoría de los autores y sobre la que, de una manera u otra, se han apoyado buena parte de los estudios sociologicos que analizan «dispositivos». Estamos hablando, por supuesto, del trabajo de Michel Foucault. ${ }^{12}$

Pretendo, por tanto, repasar los trabajos de Foucault con el objetivo de ofrecer una referencia teórica a los sociólogos que han trabajado sobre un concepto más utilizado que interrogado (Jacquinot-Delaunay \& Monnoyer 1999: 10). Una advertencia: Dado que cualquier concepto, a través de la operación reductora que lo constituye ${ }^{13}$, esconde una multiplicidad que le convierte en «el punto de coincidencia, de condensación o de acumulación de sus componentes» (Deleuze $\&$ Guattari 1991: 21), no se trata de ofrecer una definición unitaria y normativa de lo que es un dispositivo, sino de hacer visible la multiplicidad de elementos que remiten al dispositivo. El lector que busque en este artículo una definición clara y concisa de lo qué Foucault entiende por dispositivo quedará probablemente

7 «D'approches réellement réflexives, abstraites de la notion de dispositif. Le plus souvent, le terme [...] apparaît sans vraie définition, modestement lové au caur d'une phrase, d'une expression plus voyante».

8 «Comme si son caractère d'opérativité dispensait d'en penser la pertinence théorique».

9 Aunque la definición foucaultiana de dispositivo haya sido la más influyente en el campo de las ciencias sociales, lo cierto es que el término comenzo a utilizarse en la primera mitad de la década de los setenta de manera autónoma en diferentes campos. Probablemente el primero en usarlo fue Pierre Schaeffer quien, en 1971, refiriéndose a la televisión habló del dispositivo como «une piège tendu à l'animal humain pour sa capture en vue d'observation» (Schaeffer 1971: 158). De manera independiente, en el campo del análisis cinematográfico Jean-Louis Baudry utilizo el término en 1975 para definir el cine como una máquina de dominación simbolica (Baudry 1975).

10 «Permet d' exprimer de façon élégante et concise ce qui, autrement, demanderait l' emploi de périphrases hasardeuses».

${ }^{11}$ Como, por ejemplo, ha hecho por ejemplo Guy Lochard con respecto al uso del concepto en el campo de los estudios televisivos (Lochard 1999).

${ }^{12}$ La mayoría de quienes han trabajado sobre dispositivo(s) reconocen el carácter de referencia teórica fundamental (y casi única) del trabajo de Michel Foucault: Deleuze 1989, Jacquinot-Delaunay \& Monnoyer 1999, Peeters \& Charlier 1999, Berten 1999, Hert 1999, Belin 1999, Chartier 1999.

13 «Lo particular para el concepto es aquello a lo que no llega, lo que el mecanismo de su abstracción elimina, lo que no es de antemano un caso de concepto» (Adorno 1966: 16). 
decepcionado. En su lugar se encontrará con una referencia teórica, un puzzle de retales y de sugerencias.

\section{EL EMBRIÓN DE UN CONCEPTO: LA ÉPISTÉMÈ}

Para responder a la pregunta que da título a este artículo es necesario profundizar en un concepto sobre el que Foucault trabajó en los años sesenta y que no sólo puede considerarse la matriz del dispositivo ${ }^{14}$ sino un tipo de dispositivo en sí mismo ${ }^{15}$ : la épistémè. Dada la abundante bibliografía al respecto ${ }^{16}$, me limitaré aquí a dos cuestiones: esbozar una breve historia de la épistémè que permita comprender sus características fundamentales y mostrar su influencia sobre el término dispositivo.

La épistémè foucaultiana toma cuerpo en un período muy concreto de la trayectoria del filósofo (la segunda mitad de los años sesenta) en el que su preocupación fundamental giró en torno a una cuestión central: ¿Qué es el saber? ${ }^{17}$ Para dar respuesta a esta pregunta, Foucault trabaja con varios conceptos que van a llevarle primero hasta la definición de épistémè y, más adelante, hasta el dispositivo.

El primero es el de système (sistema) o «conjunto de relaciones que se mantienen, se transforman, independientemente de las cosas que ligan» ${ }^{18}$. La definición de système está muy próxima al concepto de structure (estructura) y a los trabajos de Dumèzil, Lévi-Strauss, Lacan, etc. La idea es que, «antes de toda existencia humana, de todo pensamiento humano, existiría ya un saber, un sistema que nosotros redescubrimos» ${ }^{19}$. Por tanto, ya se encuentra aquí una idea que más tarde será

14 «En el trabajo posterior de Foucault, el concepto épistémè tiende a ser remplazado por el término DISPOSITIVO» (Macey 2001: 113).

15 Idea subrayada por el propio Foucault: «Lo que llamo dispositivo es un caso mucho más general de la épistémè. O mejor que la épistémè es un dispositivo específicamente discursivo, en lo que se diferencia del dispositivo que puede ser discursivo o no discursivo, al ser sus elementos mucho más heterogéneos» (Foucault 1977: 131).

16 Para una bibliografía básica del concepto épistémè: Canguilhem 1967, Steiner 1971: 400, Lemert \& Gillan 1982: 131, Rajchman 1985: 25, Machado 1989, Sauquillo 1989: 237, Ingram 1994: 232, Macey 2001: 113.

17 «Nosotros estamos hoy en la época del saber. Normalmente, se habla de un empobrecimiento del pensamiento filosbfico, juicio inspirado por conceptos obsoletos. Hoy, hay una reflexion filos6fica extremadamente rica en un campo que no formaba parte antes de la reflexión filosofica. Los etnólogos, los lingüistas, los sociólogos, los psicólogos acometen actos filosófícos. El saber se ha diversificado, El problema filosofico contemporáneo es rodear el saber en el extremo de sí mismo, definir su propio perímetro» («Nous sommes aujourd'hui à l'âge du savoir. On parle couramment d'un appauvrissement de la pensée philosophique ; jugement inspiré par des concepts dépassés. Il y a aujourd' hui une réflexion philosophique extrêmement riche dans un champ qui ne faisait pas partie auparavant de la réflexion philosophique. Les ethnologues, les linguistes, les sociologues, les psychologues commettent des actes philosophiques. Le savoir s'est démultiplié. Le problème philosophique contemporaine est de cerner le savoir à l'extrême de lui-même, de définir son propre périmètre»), en: Foucault 1966, 580.

18 «Ensemble de relations qui se maintiennent, se transforment, indépendamment des choses qu'elles relient» (Foucault 1966b: 542).

19 «Avant toute existence humaine, toute pens6e humaine, il y aurait dejà un savoir, un système, que nous redécouvrons», en: Idem, p. 543. 
fundamental en la definición que Foucault aporta del «dispositivo disciplinario»: El sistema (igual que el dispositivo) se define a partir de un criterio de posición (Deleuze 1973: 571) que afirma que los elementos que lo componen no son significantes en sí mismos sino que su significado deriva de su posición relativa dentro del conjunto. Por otro lado, el sistema tiene un carácter constrictor que nos impide escapar de su red: siempre se piensa en el interior de una ordenación definida por una época y por un lenguaje. En este sentido, nos antecede. En 1966, Foucault definía su tarea como «poner al día este pensamiento anterior al pensamiento, ese sistema anterior a todo sistema... Él es el fondo sobre el cual nuestro pensamiento «libre» emerge y centellea durante un instante...» ${ }^{20}$.

Sin embargo, la idea de système tenía una serie de connotaciones que no convencían a Michel Foucault. La más importante, su evidente conexión con el estructuralismo ${ }^{21}$. Tal vez por ello, Foucault prefirió construir su concepto de épistémè sobre dos ideas distintas: le préalable (lo previo) y la région intermédiaire (la región intermedia).

La primera, le préalable, hace referencia a un campo de posibilidad definido por un entramado de relaciones: «Se trata de los elementos que deben haber sido formados por una práctica discursiva para que eventualmente un discurso científico se constituya, especificado no solamente por su forma y por su rigor, sino también por los objetos con los cuales tiene relación, los tipos de enunciación que pone en juego, los conceptos que manipula y las estrategias que utiliza» 22 (Foucault 1969: 237).

20 «C'est de mettre au jour cette pensée d'avant la pensée, ce système d'avant tout système...II est le fond sur lequel notre pensée «libre» émerge et scintille pendant un instant...» (Foucault 1966b: 543).

${ }^{21}$ A partir de 1968 Foucault rechazó (violentamente) cualquier vínculo con el estructuralismo: «Si preguntamos a los que han sido clasificados bajo la etiqueta de «estructuralistas», si preguntamos a Lévi-Strauss, a Lacan, a Althusser o a los lingüistas responderán que nada tienen en común unos con otros o muy pocas cosas. El estructuralismo es una categoría que existe para los otros, para quienes no lo son. Sólo desde el exterior se puede decir este o ese son estructuralistas. Es a Sartre a quien hay que preguntar qué son los estructuralistas puesto que el considera que constituyen un grupo coherente (Lévi-Strauss, Althusser, Dumézil, Lacan y yo), un grupo que presenta una especie de unidad; sin embargo esta unidad, nosotros no la percibimos.» (Foucault 1968: 42-43). Sin embargo, antes de esa fecha existen documentos donde Foucault vincula claramente su proyecto filosófico con el estructuralismo: «Lo que he intentado hacer ha sido introducir los análisis de estilo estructuralista en los dominios en los cuáles ellos no habían penetrado hasta el presente, es decir en el dominio de la historia de las ideas, la historia de los conocimientos, la historia de la teoría» («Ce que j'ai essayé de faire, c'est introduire des analyses de style structuraliste dans des domaines oi elles n'avaient pas pénétré jusqu'à présent, c'est-a-dire dans le domaine des histoires des idées. l' histoire des connaissances, l' histoire de la théorie») (Foucault 1967b: 611) En la misma entrevista, Foucault afirma: «Yo soy, a lo más, el monaguillo del estructuralismo. Digamos que yo he tocado la campana, que los fieles se han arrodillado, que los no creyentes se han puesto a gritar. Pero el oficio había comenzado hacía mucho tiempo.» («Je suis tout au plus l'enfant de chœur du structuralisme. Disons que j'ai secoué la sonnette, que les fideles se sont agenouillés, que les incroyants ont poussé des cris. Mais l'office avait commencé depuis longtemps") en: Idem, p. 609.

${ }_{22}$ «Il s'agit des éléments qui doivent avoir été formés par une pratique discursive pour qu'eventuellement un discours scientifique se constitue, spécifié non seulement par sa forme et sa rigueur, mais aussi par les objets auxquels il a affaire, les types d'énonciation qu'il met en jeu, les concepts qu'il manipule, et les stratégies qu'il utilise.» 
La segunda, la région intermédiaire, nos lleva directamente al prefacio de Les mots et les choses (1967). Allí, Foucault comienza su libro con una clasificación de animales procedente del relato de Borges «El idioma analítico de John Wilkins»: «Los animales se dividen en (a) pertenecientes al Emperador, (b) embalsamados, (c) amaestrados, (d) lechones, (e) sirenas, (f) fabulosos, (g) perros sueltos, (h) incluidos en esta clasificación, (i) que se agitan como locos, (j) innumerables, (k) dibujados con un pincel finísimo de pelo de camello, (l) etcétera, (m) que acaban de romper el jarrón, (n) que de lejos parecen moscas» ( Borges 1960: 142). Como cualquier lector, Foucault se pregunta por lo extraño de esta clasificación. ¿Por qué este conjunto nos resulta absurdo e incomprensible? Respuesta: Porque está situado en el límite de nuestro pensamiento o, más exactamente, porque es imposible de pensar. Por tanto, la pregunta clave es: «¿qué es imposible de pensar y de qué imposibilidad se trata?» ${ }^{23}$ (Foucault 1967: 1) o, a la inversa, ¿qué podemos pensar? De este modo, Foucault trata de definir el campo de lo pensable, i.e. el espacio del saber.

Foucault define dicho espacio a partir de dos puntos de referencia. En primer lugar lo que denomina «los códigos fundamentales de una cultura» o conjunto de elementos que determinan para cada hombre los órdenes empíricos a los que habrá de enfrentarse: hablamos del lenguaje, los esquemas perceptivos, las jerarquías, etc. En el otro extremo de este espacio, Foucault sitúa a las ciencias y a la fílosofía como el aparato reflexivo que explica la existencia de un orden y las leyes a las que dicho orden obedece. Entre estos dos polos, se extiende un dominio que Foucault denomina «la región intermedia». El espacio que incluye dicha región así como los dos extremos que la limitan (los códigos ordenadores y las reflexiones sobre el orden) constituye el saber. De este modo, en Les mots et les choses, Foucault propone una primera definición de épistémè ${ }^{24}$ («En una cultura y en un momento dado, siempre existe sólo una épistémè que define las condiciones de posibilidad de todo saber», Foucault 1967: 179) ${ }^{25}$ que, como bien han señalado sus críticos, estaba todavía sujeta a la acusación de estructuralismo ${ }^{26}$.

Quizá por ello sea preferible recoger la definición que aparece en $L^{\prime}$ Archéologie du savoir (1969). Allí, la épistémè es «el conjunto de relaciones que se pueden descubrir, para una época dada, entre las ciencias cuando se las analiza al

${ }^{23}$ «Qu'est-il donc impossible de penser, et de quelle impossibilité s'agit-il ?»

24 En Las palabras y las cosas, Foucault habla de tres épistémè que se han sucedido en Occidente a partir del siglo XV: (a) El Renacimiento, donde el saber está determinado por la figura de la semejanza que ordena y regula todo conocimiento posible, (b) La Edad Clásica (siglos XVII y XviII), definida por la mathesis universalis (ciencia de la medida y del orden) y por la teoría de la representación, (c) La Modernidad, que comienza a principios del siglo XIX con la desaparición de la teoría de la representación como fundamento del orden y la aparición del hombre como sujeto/ objeto del saber.

${ }_{25}$ «Dans une culture et d̀ un moment donné, il n'y a jamais qu' une épistémè, qui définit les conditions de possibilité de tout savoir».

${ }^{26}$ «Foucault admite que en Las palabras y las cosas empleó el término de un modo demasiado formal, tanto que le libro estaba abierto a la acusación de ser estructuralista» ( «Foucault admits that in The Order of the Things he employed the term in a somewhat too formal manner, such that the book was open to the charge of being structuralism»), en (Gillan \& Lemert 1982: 131) Ver también: (Barker 1993: 53). 
nivel de sus regularidades discursivas» ${ }^{27}$ (Foucault 1969: 250). Como el propio Foucault señala, la épistémè no es ni el estilo general de las investigaciones en un período concreto, ni una cierta mentalidad que pudiera subyacer a todas ellas, ni una determinada visión del mundo (no es die Weltanschauung), ni una estructura general del pensamiento. Todas estas aproximaciones se refieren a lo unitario. La épistémè, por el contrario, describe un conjunto de relaciones, una dispersión. No se trata de un estadio de racionalidad que atraviesa cualquier forma del conocimiento en una época dada, tampoco de la manifestación última de la inquebrantable unidad del sujeto, «sino de la desviación, las distancias, las oposiciones, las diferencias, las relaciones de sus múltiples discursos científicos.» (Foucault 1968b: 50)

En definitiva, tomando como referencia esta definición y como veremos más adelante, puede señalarse una doble coincidencia entre el concepto de épistémè y el de dispositivo:

- El primer lugar, ambos remiten a un espacio topológico, i.e. un espacio que se define tanto por la posición que ocupan los elementos que se distribuyen en él (incluida la distancia que los separa) como por las funciones de dichos elementos. Como veremos más adelante, los sociólogos han utilizado recurrentemente esta idea para definir «dispositivos». Un buen ejemplo es el «dispositivo pedagógico» donde sus elementos (el profesor, los alumnos, etc.) no son significantes per se, sino que adquieren su significado por la posición que ocupan en un determinado espacio, por la función que desempeñan y por el tipo de relaciones que entablan entre ellos.

- En segundo lugar, ambos se refieren a una multiplicidad. Tanto la épistémè como el dispositivo definen multiplicidades de elementos: la primera hace referencia a la pluralidad de componentes del espacio del saber, el segundo a un conjunto de piezas que, en forma de réseau, estructuran un espacio determinado.

\section{EL DISPOSITIVO}

A través de un movimiento que «subraya el tránsito del momento de la «arqueología» al propio de la «genealogía»,»(Sauquillo 1989: 241) a mediados de la década de los setenta Foucault reemplaza el concepto de épistémè por el de dispositivo. En este sentido, los dos libros claves son Surveiller et punir (1975) ${ }^{28}$ y La volonté de savoir (1976) ${ }^{29}$. Con vistas a comprender el uso que Foucault

${ }^{27}$ «L' ensemble des relations qu'on peut découvrir, pour une époque donnée, entre les sciences, quand on les analyse au niveau des régularités discursives".

${ }^{28}$ Hay traducción castellana: FouCAULT, M. 1992. Vigilar y castigar. Nacimiento de la prisión, S. XXI, Madrid.

${ }^{29}$ Hay traducción castellana: Foucault, M. 1984. La historia de la sexualidad I. La voluntad de saber, Siglo XXI, Madrid. 
hace de este concepto procederemos en dos tiempos: En primer lugar, ofreceremos algunos ejemplos concretos (la definición del «examen» en tanto que dispositivo disciplinario) y en segundo lugar esbozaremos un análisis teórico de la noción foucaultiana de dispositivo.

\subsection{Un ejemplo de dispositivo: el examen}

En Surveiller et punir, Foucault examina el funcionamiento del poder y habla del «dispositivo disciplinario» como el específico de nuestra contemporaneidad: «El poder disciplinario [...] se convierte en un «sistema integrado», ligado desde el interior a la economía y a los fines del dispositivo donde se ejerce» ${ }^{30}$ (Foucault 1975: 179) El dispositivo disciplinario se organiza como un poder múltiple, automático y autónomo que funciona a través de múltiples técnicas que se entrecruzan y se extienden por el tejido social en forma de redes: «Poder que no se encuentra ubicado históricamente en las instancias superiores de la censura, sino que también se sumerge más profundamente, más sutilmente, en todo el entramado de la sociedad» (Foucault 1972: 107).

Ese gran dispositivo que conforma el «aparato disciplinario» (Foucault 1975: 176) se articula sobre una serie de instrumentos que se entrecruzan en el juego de la vigilancia, del castigo y del examen. El más representativo de estos «dispositivos de disciplina» (Foucault 1975: 187) es el examen, procedimiento que combina las técnicas de la vigilancia y de la sanción normalizadora y que establece sobre los individuos una vigilancia a través de la cual les diferencia y les sanciona. Por ello, tomaremos el examen como ejemplo de la descripción que hace Foucault de un dispositivo.

El examen es la forma jurídica característica de nuestra modernidad que combina la vigilancia y el castigo, regulando en su interior un tipo de relación entre saber y poder Esta forma de saber-poder dará lugar a las «ciencias humanas» (psiquiatría, psicología, sociología, etc.) surgidas dentro del dispositivo definido por el propio examen: ciencias nacidas para clasificar, vigilar, determinar la posición exacta del individuo dentro de una sociedad ( $\mathrm{y}$, lo que es más importante, su peligrosidad). La liaison saber-poder definida por el examen se caracteriza por tres rasgos:

1. El examen invierte la economía de la visibilidad en el ejercicio del poder. Hasta el siglo XIX, el poder tiene un carácter esencialmente exhibicionista: se muestra, se manifiesta, se hace visible. El poder regula un ceremonial a través del cual se muestra como triunfo: la coronación del soberano, la rendición del vencido, el martirio del condenado, etc. Por el contrario, el dominado permanece en el anonimato, forma parte de una masa anónima cuya función es mirar. A partir del siglo XIX el poder disciplinario se despliega en su invisibilidad, sometiendo a una visibilidad obligatoria a aque-

30 «Le pouvoir disciplinaire [...] devient un système «intégré», lié de l'intérieur à l'économie et aux fins du dispositif où il s' exerce." [El subrayado es mío] 
llos sobre quienes se aplica. Con la disciplina, el poder pasa de observado a observador. Su mirada no será otra que el examen, entendido como el conjunto de técnicas encaminadas a «objetivizar» al individuo definiéndolo a partir de una serie de parámetros. La disciplina establece su propia ceremonia, a través de la cual «los «sujetos» son ofertados como «objetos» a la observación de un poder que no se manifiesta más que en su sola mirada» (Foucault 1975: 190).

2. El examen hace entrar al individuo en un campo documental. El examen, que somete a los individuos a permanente vigilancia, se fundamenta sobre el desarrollo y la acumulación de una inmensa masa documental. Siguiendo los métodos utilizados por la burocracia administrativa, el examen elabora, a partir de las diferentes instituciones que articula, numerosos informes sobre el individuo: el colegio (calificaciones académicas y sobre el comportamiento de las personas), el hospital (informes médicos), el cuartel militar. De este modo, el examen posibilita la constitución del individuo como un «objeto» descriptible dentro de un campo relacional más amplio. Ese objeto es el ciudadano moderno.

3. El examen hace de cada individuo un "caso" concreto. No hay que entender este término en su acepción jurídica, más bien se refiere «al individuo tal y como podemos describirle, evaluarle, medirle, compararle a otros y esto en su individualidad propia» ${ }^{31}$ (Foucault 1975: 193). Antes del siglo XIX, la descripción detallada de la vida de una persona (normalmente bajo la forma de biografía) era tanto un privilegio como un testigo del poder del personaje biografiado. El objetivo era conservar su memoria frente al paso de los años. Con la sociedad disciplinaria, la biografía adopta la forma de informe y la descripción de la individualidad se convierte en un mecanismo de control utilizable en el presente. De este modo surge una nueva figura (el individuo) como efecto y objeto tanto del saber como del poder: «En un régimen disciplinario, la individualización en revancha es descendente: a medida que el poder deviene más anónimo y más funcional, aquellos sobre los que se ejerce tienden a ser más fuertemente individualizados; y por las vigilancias más que por las ceremonias, por las conmemoraciones más que por los relatos conmemorativos, por las medidas comparativas que tienen la «norma» por referencia» (Foucault 1975: 195).

En definitiva, en Surveiller et punir Foucault ofrece una idea de dispositivo que va más allá de su trabajo epistemológico. Como la épistémè, el dispositivo describe el espacio de una dispersión, la realidad de una multiplicidad de elementos. Sin embargo, el dispositivo introduce nuevos elementos: (a) define una serie de conexiones íntimas entre saber y poder ${ }^{32}$ (el examen es una forma de

${ }^{31}$ «C'est l'individu tel qu'on peut le décrire, le jauger, le mesure, le comparé à des autres et cela dans son individualité même».

32 «Es necesario por tanto admitir que el poder produce saber [...] que poder y saber se implican directamente el uno al otro, que no hay relación de poder sin constitución relativa de un campo de saber, ni saber que no suponga y no constituya al mismo tiempo relaciones de poder» (Il faut 
saber y, al mismo tiempo, el ejercicio de un poder), (b) establece la dispersión del poder a través una multiplicidad de dispositivos (la vigilancia, el castigo, el examen) y (c) describe la producción de modos de subjetivación del individuo a partir de determinadas técnicas (el propio examen). Ideas que Foucault retomará más adelante en La Volonté de savoir (Foucault 1976). Allí, el dispositivo de la sexualidad hace referencia al conjunto de prácticas, instituciones y conocimientos que hicieron, hacia el siglo XVIII, de la sexualidad un dominio coherente y una dimensión absolutamente fundamental del individuo. Frente a quienes definen la sexualidad como represión, Foucault propone insertar esa hipótesis represiva en un dispositivo más amplio que permitiera comprender la sexualidad como un campo estratégico donde se ligan discursos, prácticas, tácticas, estrategias, poder-represión, poder-seducción y modos de subjetivación.

\subsection{Hacia una descripción teórica del dispositivo}

Toda vez mostrados algunos ejemplos de lo que Foucault entiende por dispositivo es el momento de proceder a una definición teórica. Aunque, en mi opinión, Deleuze hace una interpretación excesivamente amplia del término, su definición es un buen punto de partida: Un dispositivo es «una especie de ovillo o madeja, un conjunto multilineal. Está compuesto de líneas de diferente naturaleza y esas líneas del dispositivo no abarcan ni rodean sistemas cada uno de los cuáles serían homogéneos por su cuenta (el objeto, el sujeto, el lenguaje), sino que siguen direcciones diferentes, forman procesos siempre en desequilibrio y esas líneas tanto se acercan una a otras como se alejan unas de otras» (Deleuze 1989: 155) Deleuze distingue cuatro líneas principales que componen un dispositivo:

- Líneas de visibilidad. Los dispositivos tienen como primera función hacer ver. Su régimen de luz describe una arquitectura de la realidad, haciendo visibles ciertas partes y dejando otras en penumbra.

- Líneas de enunciación. Su función es hacer hablar a través de la producción de un régimen de enunciación concreto. Estas líneas determinan el espacio de lo enunciable, aquello que puede ser dicho en el campo de un dispositivo dado.

- Líneas de fuerza. Añaden la tercera dimensión que permite al dispositivo ocupar un determinado lugar en el espacio, adoptar una forma concreta. Recorren la interioridad de dicho espacio (o más bien la atraviesan) y regulan el tipo de relaciones que pueden producirse.

- Líneas de subjetivación. Se refieren al individuo y describen las condiciones en las que este se convierte en sujeto/objeto de conocimiento, defi-

plutôt admettre que le pouvoir produit du savoir [...] que pouvoir et savoir s'impliquent directement l'un l'autre ; qu'il n'y a champ des relations de pouvoir sans constitution corrélative d' un champ de savoir, ni de savoir qui ne suppose et ne constitue en même temps des relations de pouvoirm), Foucault 1975: 32. 
nen procesos y funcionan como líneas de fuga: «Escapa a las líneas anteriores, se escapa. El sí-mismo no es ni un saber ni un poder. Es proceso de individuación que tiene que ver con grupos o personas y que se sustrae a las relaciones de fuerzas establecidas como saberes constituidos: es una especie de plusvalía» (Deleuze 1989: 157).

De alguna manera, los dos primeros tipos de líneas remiten al saber y ya se encontraban presentes en la definición de épistémè. De acuerdo con Deleuze, el dispositivo incorpora dos nuevas dimensiones (referidas al poder y a los procesos de subjetivación), que dotan de volumen a la figura bidimensional de la épistémè.

Tomando como referencia la definición de Foucault (Foucault 1977: 127131), el dispositif podría representarse del siguiente modo:

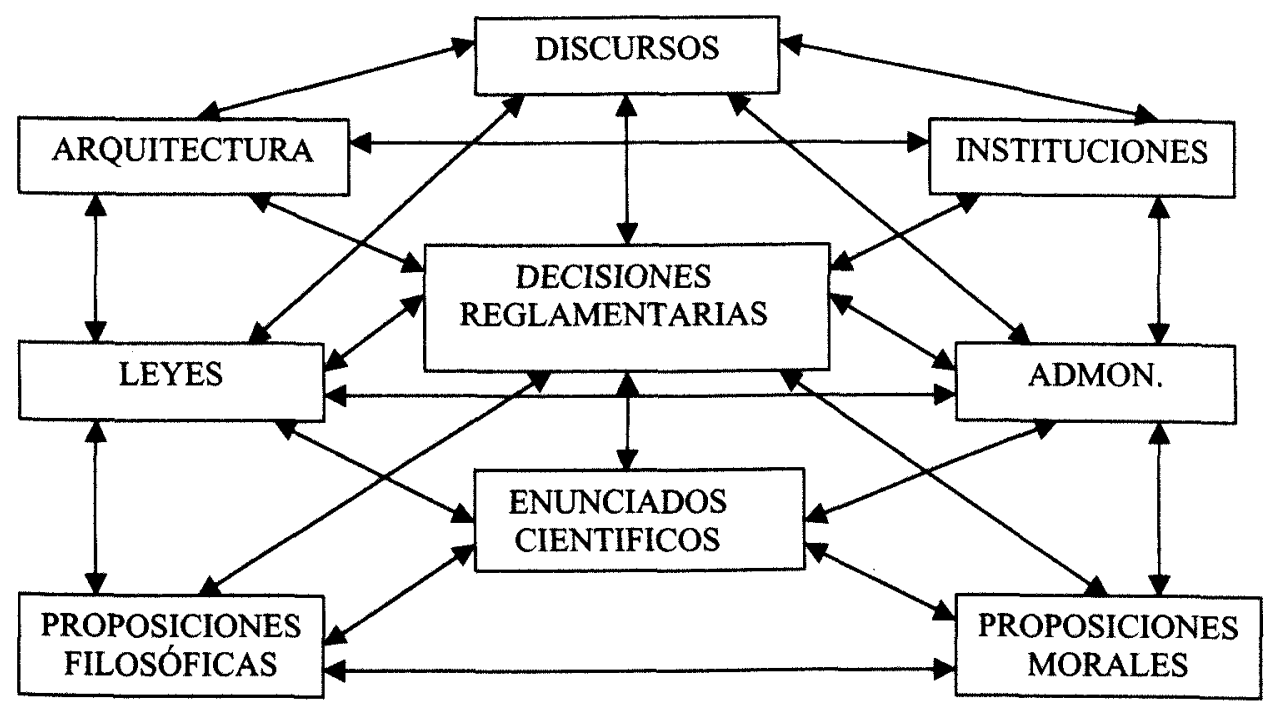

Descripción de un dispositivo.

En el esquema que acabo de esbozar, el dispositivo se define no sólo por cada uno de los elementos allí enumerados (discursos, instituciones, etc.), también, y sobre todo, por la malla que se establece entre ellos. Foucault define el dispositivo a partir de tres características: (a) como retícula o red, (b) como un tipo de relación: «Entre estos elementos, discursivos o no, existe como un juego, de los cambios de posición, de las modificaciones de funciones que pueden, éstas también, ser muy diferentes» (Foucault 1977: 129), (c) como un juego de fuerzas o, más bien, como «estrategias de relaciones de fuerzas soportando unos tipos de saber, y soportadas por ellos» (Foucault 1977: 130-131). 


\section{4. «DISPOSITIVO» Y CIENCIAS SOCIALES}

En lugar de proceder a una definición normativa de «dispositivo», hemos esparcido sobre la mesa algunas ideas desarrolladas por Foucault en torno a este meta-concepto (Nel 1999: 131): «épistémè», «espacio topológico», «multiplicidades», «relación de fuerzas», «saber-poder», «subjetividad», etc. Es este arsenal heterogéneo de imágenes, esquemas, figuras y modelos el que ha determinado la diversidad de contextos de utilización del término. Exploraremos ahora las líneas generales de este mode d'emploi en el terreno de las ciencias sociales.

El dispositivo como «esquema primordial del pensamiento» ${ }^{33}$. Foucault define el dispositivo como la red que puede establecerse entre un conjunto heterogéneo de elementos que incluye discursos, instituciones, reglamentos, leyes, medidas administrativas, enunciados científicos, proposiciones filosóficas y morales. Por tanto, el concepto hace referencia a un esquema de representación o grille d'intérpretation (cuya forma remite a una retícula o a una red) ${ }^{34}$ a través del cuál pensar fenómenos socio/ culturales. Tanto la épistéme como el dispositivo describen espacios topológicos, i.e. espacios definidos por la relación entre un conjunto heterogéneo de elementos que no son significantes en sí mismos. Así, la idea de réseau que pone en juego el «dispositivo» se encuentra a medio camino entre el concepto de «estructura» (que reduce la multiplicidad a un esquema totalizante) y el de «rizoma» (pura multiplicidad).

Esta idea recorre los trabajos sobre sociología de los medios de comunicación que han utilizado el dispositivo para «designar un campo compuesto de elementos heterogéneos y para tratar esta heterogeneidad» ${ }^{35}$ (Peeters \& Charlier 1999: 15). Así, «el dispositivo se define en una función de soporte, de baliza, de cuadro organizador» ${ }^{36}$, (Peeters \& Charlier 1999: 19) y si organiza y hace posible «algo» es porque «simplemente hace existir un espacio particular previo en el que ese «algo» puede producirse» ${ }^{37}$ (Peeters \& Charlier 1999: 19). El dispositivo puede ser entendido como el environnement (Berten 1999: 39) que da cuenta de la relación que se establece entre los seres humanos y los objetos en la socie-

33 Deleuze y Guattari denuncian como el pensamiento occidental, desde la biología a la lingüística, ha adoptado la imagen de un árbol-raíz. Esta imagen supone la existencia de «una fuerte unidad principal» sobre la que pivotan las raíces secundarias, de tal modo que «el árbol o la raíz inspiran una triste imagen del pensamiento que no cesa de imitar lo múltiple a partir de una unidad superior, de centro o de segmento» (Deleuze \& Guattari 1976: 36). Frente a ello, estos autores proponen sistemas de tipo rizoma: «Haced rizoma y no raíz, no plantéis nunca! ¡No sembréis, horadad! ¡No seáis ni uno ni múltiple, sed multiplicidades! ¡Haced la línea, no el punto! (Deleuze \& Guattari 1976: 56). Entre el árbol-raíz y el rizoma, el dispositivo se constituye como reseau.

${ }^{34} \mathrm{El}$ nacimiento del concepto de réseau como figura electiva del pensamiento moderno se remonta a los años 1820 y a Saint-Simon (Musso 1987-1988).

35 «Désigner un champ composé d'éléments hétérogènes [...] et de traiter cette hétérogénéité».

36 «Le dispositif se définit dans une fonction de support, de balise, de cadre organisateur».

${ }^{37}$ «Il fait simplement exister un espace particulier préalable dans lequel ce "quelque chose» peut se produire». 
dad contemporánea, relación que no se apoya en una radical separación de lo técnico y lo simbólico sino en la integración de ambos en un dispositivo (Berten 1999: 39). También ha sido interpretado como «una instancia, un lugar social de interacción y de cooperación» (Peraya 1999: 153) a partir del cuál analizar la utilización de tecnología en ambientes educativos. En el campo de la sociología del trabajo, el dispositivo ha sido descrito como un «espacio de efectividad» ${ }^{38}$ (Fusulier \& Lannoy 1999: 190) cuya fuerza reside en su capacidad para conciliar una efectividad plural: la de los gestores que esperan alcanzar los objetivos fijados y la de los múltiples usuarios. No se trata por tanto ni de un aparato ni de un mercado, sino como un espacio híbrido. (Fusulier \& Lannoy 1999: 190). En la misma línea, Eymard-Duvernay habla de «dispositivos» como «conjunto de rutinas incorporadas y de objetos que condicionan su uso por los actores» ${ }^{39}$ (Eymard-Duvernay 1997: 17)

Valgan estos ejemplos para mostrar como, en el campo de las ciencias sociales, la idea foucaultiana del dispositivo como un réseau ha abierto nuevas posibilidades frente a otros tipos de imaginarios teóricos.

En segundo lugar, la noción foucaultiana de dispositivo planteaba (como hemos visto en el caso del examen) la existencia de procesos de subjetivación a través de los cuáles el individuo se convierte en sujeto. En definitiva, y rompiendo con la idea de que el hombre y el mundo son dos entidades separadas, el dispositivo se ha convertido en una referencia a través de la cual examinar los procesos de subjetivación que determinan la vida de las personas. Así por ejemplo, André Berten (Berten 1999: 36-37) considera dos modalidades de dispositivos: (a) Los dispositivos sociales, políticos y económicos que dan forma el individuo, le orientan, le inculcan un determinado saber y le atribuyen un cierto poder, y (b) Los dispositivos psicológicos, morales, reflexivos que el individuo se da a sí mismo para formarse, conocerse, orientarse. (Berten 1999: 36-37).

Esta idea ha sido fundamental en el campo de la sociología del trabajo donde se ha replanteado la relación entre el hombre y las máquinas. Así, «la noción de dispositivo [...] contribuye a la reformulación de una problemática antigua y recurrente que es la del status de los objetos técnicos, la del uso y de apropiación de los útiles en las relaciones hombres-máquinas» ${ }^{40}$ (JacquinotDelaunay \& Monnoyer 1999: 11). Dicho concepto ha posibilitado un modelo alternativo de acción donde el actor ya no se define por constituir una entidad separada de las máquinas sino por compartir con ellas una relación en el marco de un dispositivo. De este modo, los dispositivos técnicos concebidos como «un compuesto de humano y de no humano» ${ }^{41}$ (Peeters \& Charlier 1999: 17) son fundamentales para comprender la experiencia del hombre moderno. En este sentido y dado que en las sociedades modernas el individuo se constituye

\footnotetext{
38 «Espace d' effectivité».

39 «Ensemble de routines incorporées et d'objets qui conditionnent son usage par les acteurs».

40 «La notion de dispositif (...) contribue à la reformulation d'une problématique ancienne et récurrente qui est celle du status des objets techniques, de l'usage et de l'appropriation des outils».

41 «Un composé d' humain et de non-humain*.
} 
como sujeto a través de sus relaciones con las máquinas, algunos sociólogos han hablado de la necesidad de valorizar los objetos y las relaciones que tenemos con ellos (Tisseron 1999: 57): «El concepto de dispositivo parece dar cuenta del hecho de que una nueva relación con los objetos caracteriza a la sociedad contemporánea o de que otra relación con el mundo material, de los objetos, es posible, no según el modo de la instrumentación o de la alineación, sino a través de la frecuentación, el contacto o incluso la experiencia afectivocorporal» (Peeters \& Charlier 1999: 17) ${ }^{42}$.

Por último, hay que señalar que la sociología de las ciencias ha extraído al menos dos enseñanzas de la definición foucaultiana de dispositivo. En primer lugar la necesidad de analizar prácticas concretas: «Analizar el saber y el poder que están haciéndose, como diría Latour, que están construyéndose; atraer nuestra atención sobre el aspecto productivo de un cierto número de dispositivos» ${ }^{43}$ (Berten 1999: 35) En segundo lugar, una manera diferente de comprender la relación entre saber y poder: «No solamente cada dispositivo incluye saberes múltiples, transversales, ramificados, sino que el propio dispositivo se convierte en un medio productor de saber» ${ }^{44}$ (Berten 1999: 35). A través del análisis de la praxis científica, el «dispositivo» introduce una idea fundamental en el campo de la sociología de la ciencia: El poder produce saber. Frente a la tradición que desde Platón establece la incompatibilidad entre ambos ${ }^{45}$, Foucault recupera el pensamiento nietzscheano para mostrar que «el poder produce saber [...] que poder y saber se implican directamente el uno al otro, que no existe relación de poder sin constitución relativa de un campo de saber, ni saber que no suponga y no constituya al mismo tiempo unas relaciones de poder» ${ }^{46}$ (Foucault 1975: 32).

${ }^{42}$ «Le concept de dispositif semble rendre compte du fait qu' un nouveau rapport aux objets caractérise la société contemporaine ou qu' un autre rapport avec le monde matériel, objectal est possible, non plus sur le mode d'instrumentation ou d'aliénation, mais sur le mode de la fréquentation, du contact ou même de l'expérience affectivo-corporelle».

${ }^{43}$ «Analyser le savoir et le pouvoir en train de se faire, comme dirait Latour, en train de se construire, attire notre attention sur l'aspect productif d' un certain nombre de dispositifs".

44 «Non seulement chaque dispositif inclut des savoirs multiples, transversaux, ramifiés, mais encore, le dispositif lui-même devient un milieu producteur de savoir».

${ }_{45}$ En opinión de Foucault, dicha tradición nace en Grecia en el momento en que, entre el modelo sofista y platónico, se optó por el segundo. Los sofistas interpretaban la constitución de un determinado saber como indisociable del ejercicio del poder, pero dicha concepción fue desterrada por el triunfo del platonismo y por la idea de que saber y poder son incompatibles: «Se puede decir que, siguiendo a Platón, toda la filosofía de Occidente consistio en establecer el máximo de distancia entre uno y otro. Esto dio lugar a los temas, por una parte, de la idealidad del saber, pero también dio lugar a otra muy curiosa y muy hipócrita división del trabajo entre los hombres de poder y los hombres de saber, dio lugar a este curioso personaje, el del sabio, el científico que debe renunciar a cualquier poder, renunciar a cualquier participación en la ciudad, para adquirir la verdad. Todo esto constituye la fábula que Occidente se cuenta a si mismo para enmascarar su sed, su gigantesco apetito de poder sirviéndose del saber» (Foucault 1973: 155).

46 «Le pouvoir produit du savoir [...] ; que pouvoir et savoir s'impliquent directement l'un l'autre; qu'il n'y a pas de relation de pouvoir sans constitution corrélative d'un champ de pouvoir, ni de savoir qui ne suppose et ne constitue en même temps des relations de pouvoir». 
Esa idea no sólo ha articulado la sociología de la ciencia al estilo de Hacking o de Latour, sino que ha posibilitado trabajos sobre la televisión como un dispositivo de vigilancia social (Allemand 1980 y 1983, Bourdon 1994, Nel 1998 y 1999), sobre el cine como dispositivo de dominación simbólica (Baudry 1975) o sobre los dispositivos escolares como una realidad heterogénea donde se mezclan textos oficiales y reglamentos, aulas y mobiliario, alumnos y profesores en un juego de saber-poder (Chartier 1999).

\section{A MODO DE CONCLUSIÓN}

Este artículo trata de superar lo que podría llamarse la paradoja del dispositivo, paradoja que resulta de la prolífica utilización del término en numerosos campos de la investigación social durante los últimos años y de los escasos ejercicios de reflexión teórica que ha suscitado.

Dicha situación provoca el evidente riesgo de vaciar el concepto de significado. El «dispositivo» corre el peligro de seguir el mismo camino que otros conceptos como el de «postmodernismo» del que decía Richard Rorty que, después de tantas páginas dedicadas a su definición, se había convertido en un concepto demasiado borroso para transmitir nada. (Rorty 2000: 17). Conviene reiterar que dicha amenaza no remite a un problema teórico sino práctico: desde hace algún tiempo son varios los trabajos que hacen una utilización inadecuada y descontextualizada del concepto precisamente por haber renunciado a la siempre complicada (pero necesaria) tarea de dotarlo de contenido.

De cualquier modo, no pretendíamos aquí ofrecer la única definición posible del concepto. Tal y como hemos tratado de mostrar, existen otras posibilidades de aproximación teórica que deben ser exploradas y que deben enriquecer la reflexión sobre el mismo. En este sentido, hemos optado por una de las opciones posibles: retomar la reflexión foucaultiana del «dispositivo» (reflexión que, a nadie se le escapa, está en el origen de su amplia difusión) y mostrar su influencia sobre numerosos trabajos que se enmarcan dentro de una metodología cualitativa de las ciencias sociales. Esperamos que dicha reflexión pueda convertirse tanto en una referencia válida para futuros trabajos empíricos como en una invitación a quienes, desde el campo de la historia de las ideas, deben retomar el desafío lanzado por Deleuze hace casi quince años y respondiendo a una pregunta de plena actualidad: ¿Qué es un dispositivo?

\section{REFERENCIAS BIBLIOGRÁFICAS}

Allemand, E. (1983): Pouvoir et télévision. Les machines d'organisation, Paris: Anthropos.

- (1983): L'information scientifique d la télevision, Paris: Anthropos.

ADORNo, T. W. (1966): Dialéctica negativa, Madrid: Taurus, 1989. 
BARKER, P. (1993): Michel Foucault. Subversions of the Subject, Hertfordshire: Harvester Wheatsheaf.

BAUDRY, J. L. (1975): «Le dispositif: approches métapsychologiques de l'impression de la réalité», Communications, n. $^{\circ} 23$, pp. 56-72.

BELIN, E. (1999): «De la bienveillance dispositive», Hermès. Cognition, communication, politique, $\mathrm{n}^{\circ} 25,1999$, pp. $245-259$.

BERTEN, A. (1999): «Dispositif, médiation, créativité: petite généalogie», Hermès. Cognition, communication, politique, ${ }^{\circ}{ }^{\circ} 25$, pp. 33-47.

BESSY, C. (1997): «Les dispositifs d'évaluation des compétences. Une approche statistique par branche d'activité», en: C. Bessy \& F. Eymard-Duvernay (1997): Les intermédiaires du marché du travail, Paris: Presses Universitaires de France, pp. 35-51.

BORGES, J. L. (1960): Otras inquisiciones, Buenos Aires: Emecé Editores.

Bourdon, J. (1994): Haute fidélité: pouvoir et télévision. 1935-1994. Paris: Seuil.

CANGuILhem, G. (1967): «Mort de l'Homme ou Épuisement du Cogito ? Review of Foucault's Les mots et les choses", en: B. Smart (ed.). 1994. Michel Foucault. Critical Assessments. Volume 1, London: Routledge, pp. 355-368.

CARDON, D. (1997): «La coopération dans les situations de travail», Présentation, Réseaux, Paris: CENT/ CNRS.

CHARTIER, A. M. (1999): «Un dispositif sans auteur: cahier et classeurs à l'école primaire», Hermès. Cognition, communication, politique, n. ${ }^{\circ} 25,1999$, pp. 207-218.

Deleuze, G. (1973): «¿En qué se reconoce el estructuralismo?», en: F. Châtelet (dir.): Historia de la filosofía. Tomo IV, Madrid: Espasa Calpe, 1976, pp. 567-599.

- \& GuATTARI, F. (1976): Rizoma (Introducción), Valencia: Pre-textos, 2000.

- (1989): «¿Qué es un dispositivo?», en: Michel Foucault, filósofo, Gedisa, Barcelona: Gedisa, pp. 155-163.

- \& GuatTARI, F. (1991): ¿Qué es filosofía?, Barcelona: Anagrama, 1993.

EYMARD-DUVERNAY, F. (1997): «Les contrats de travail: une approche comparative», en: C. Bessy \& F. Eymard-Duvernay (1997): Les intermédiaires du marché du travail, Paris: Presses Universitaires de France, pp. 3-33.

FlaGeul, A. (1999): «Televisión: L'Age d'or des dispositifs. 1969-1983», Hermès. Cognition, communication, politique, $\mathrm{n} .^{\circ} 25,1999, \mathrm{pp} .123-130$.

Foucault, M. (1966): «Michel Foucault et Gilles Deleuze veulent rendre à Nietzsche son vrai visage», Dits et écrits I, 1954-1975, Paris: Gallimard, 2001, pp. 577-580.

- (1966b): «Entretien avec Madeleine Chapsal», Dits et écrits 1, 1954-1975, Paris: Gallimard, 2001, pp. 541-546.

- (1967): Les mots et les choses. Une archéologie des sciences humaines, Paris: Gallimard.

- (1967b): «La philosophie structuraliste permet de diagnostiquer ce qu'est «aujourd' hui», Dits et écrits I, 1954-1975, Paris: Gallimard, 2001, pp. 608-612.

- (1968): «Foucault responde a Sartre», en: Saber y verdad, Madrid: Las Ediciones de la Piqueta, 1991, pp. 39-46.

- (1968b): «La función política del intelectual. Respuesta a una cuestión.», en: Saber y verdad, Madrid: Las Ediciones de la Piqueta, 1991, pp. 47-74.

- (1969): L'Archéologie du savoir, Paris: Gallimard.

- (1972): «Los intelectuales y el poder»,en: Estrategias de poder. Obras esenciales. Volumen II, Barcelona: Paidós, 1999, pp. 106-115.

- (1973): «De la arqueología a la dinástica», en: Estrategias de poder. Obras esenciales. Volumen II, Barcelona: Paidós, pp. 145-157.

- (1975): Surveiller et punir. Naissance de la prison, Gallimard, Paris: Gallimard.

- (1976): La volonté de savoir, Paris: Gallimard. 
- (1977): «El juego de Michel Foucault», en: Saber y verdad, Madrid: Las Ediciones de la Piqueta, 1991, pp. 127-162.

FusulieR, B. \& LANNOY, P. (1999): «Comment “aménager par le management"», Hermès. Cognition, communication, politique, n. ${ }^{\circ} 25,1999$, pp. 181-197.

HERT, P. (1999): «Internet comme dispositif hétéretopique», Hermès. Cognition, communication, politique, $\mathrm{n}^{\circ} 25,1999, \mathrm{pp} .93-107$.

INGRAM, D. (1994) «Foucault and Habermas on the subject of reason», en: G. Gutting (ed.): The Cambridge Companion to Foucault, Cambridge: Cambridge University Press, 215-261.

JACQUinot-Delaunay, G. \& Monnoyer, L. (1999): "Avant-propos», Hermès. Cognition, communication, politique, $\mathrm{n} .^{\circ} 25,1999$, pp. 9-14.

LATOUR, B. \& WOOLGar, S. (1979): Laboratory Life. The Social Construction of Scientific Facts, California: Sage Publications.

- (1987): Science in action. How to follow scientist and engineers through society, Strattford: Open University Press.

Lemert, Charles C. \& Gillan, G. (1982): Michel Foucault. Social Theory as Transgression. New York: Columbia University Press.

LochaRD, G. (1999): «Parcours d'un concept dans les études télévisuelles», Hermès. Cognition, communication, politique, n. ${ }^{\circ} 25$, pp. 143-151.

MACEY, D. (2001): The Penguin Dictionary of Critical Theory, London: Penguin Books.

MACHADO, R. (1989): «Arqueología y Epistemología», en: Michel Foucault, Filósofo. (Encuentro Internacional organizado en París en enero de 1988 por la Association pour le Centre Michel Foucault), Barcelona: Gedisa, 1990, pp. 15-30;

Musso, P. (1988-1989): "Aux origines du concept moderne: l'organisme et le réseau dans la physiologie de Saint-Simon,» Quaderni, 3, pp. 11-29.

NeL, N. (1998): «Les dispositifs télévisuels», en: J. Bourdon \& F. Jost (dir.), Penser la télévision, Paris: INA-Nathan, pp. 59-71.

- (1999): «Des dispositifs aux agencements télévisuels», Hermès. Cognition, communication, politique, $\mathrm{n}^{\circ} 25$, pp. 131-141.

Peeters, H. \& Charlier, P. (1999): «Contributions à une théorie du dispositif», Hermès. Cognition, communication, politique, $\mathrm{n} .^{\circ} 25,1999, \mathrm{pp} .15-23$.

PerayA, D. (1999): «Médiation et médiatisation: le campus virtuel», Hermès. Cognition, communication, politique, $\mathrm{n}^{\circ} 25,1999, \mathrm{pp} .153-167$.

Porrou, J. P. (1980): «Critique de la théorie de l'habitus», Technologies, Idéologies, Pratiques, 2 (1), pp. 71-97.

Rajchman, J. (1985): Michel Foucault. The freedom of philosophy, New York: Columbia University Press.

RORTY, R. (2000): «Pragmatismo, pluralismo y postmodernismo», ENDOXA: Series Filosóficas, n. ${ }^{\circ} 12,2000$, UNED, Madrid.

SAUQuILlo GonzÁlez, J. (1989): Michel Foucault: una filosofía de la acción, Madrid: Centro de Estudios Constitucionales.

SChaeffer, P. (1971): Machines à communiquer. Tome Il. Pouvoir et communication, Paris: Le Seuil.

Steiner, G. (1971): «The Order of Things: Review of Michel Foucault's An Archaeology of the Human Sciences», en: B. Smart (ed.). 1994. Michel Foucault. Critical Assessments. Volume I, London: Routledge, pp. 398-403.

Tisseron, S. (1999): «Nos objets quotidiens», Hermès. Cognition, communication, politique, ${ }^{\circ}{ }^{\circ} 25,1999$, pp. 57-66.

VANDENDORPE, F. (1999): «Un cadre plus normatif qu'il n'y paraît: les pratiques funéraires», Hermès. Cognition, communication, politique, n. ${ }^{\circ} 25$, pp. 199-205. 


\section{RESUMEN}

En los áltimos años hemos asistido a una multiplicación del número de trabajos que plantean la existencia de diversos «dispositivos» en el campo de las ciencias sociales. Teniendo en cuenta los escasos ejercicios de reflexión teórica sobre dicho concepto, este artículo repasa la referencia a la que dichos trabajos remiten: la definición de Michel Foucault. El objetivo de esta reflexión es doble: Por un lado, ofrecer a los sociólogos un enfoque reflexivo sobre un concepto muy utilizado pero raramente definido, y por otro, mostrar como el término «dispositivo» se ha convertido en una referencia clave para la metodología de las ciencias sociales.

\section{ABSTRACT}

In recent years, there has been an increase in the number of works which focus on the diverse «dispositifs» in the social sciences. Given the small number of works which reflect critically on this concept, this article revisits the original definition by Michel Foucault. It will, on the one hand, offer a critical perspective of a commonly used but seldom defined term, and on the other, outline how the term «dispositifs» has been converted into a key reference for the methodology of the social sciences. 